\title{
Marital status and survival of patients with oral cavity squamous cell carcinoma: a population-based study
}

\author{
Xiao Shi ${ }^{1,2}$, Ting-ting Zhang ${ }^{1,2}$, Wei-ping $\mathrm{Hu}^{3}$, Qing-hai $\mathrm{Ji}^{1,2}$ \\ ${ }^{1}$ Department of Head and Neck Surgery, Fudan University Shanghai Cancer Center, Shanghai, China \\ ${ }^{2}$ Department of Oncology, Shanghai Medical College, Fudan University, Shanghai, China \\ ${ }^{3}$ Department of Respiratory Medicine, Zhongshan Hospital, Fudan University, Shanghai, China
}

Correspondence to: Qing-hai Ji, email: jiqinghai@shca.org.cn

Keywords: oral cavity squamous cell carcinoma, marital status, SEER, survival analysis, spousal support

Received: November 04, 2016

Accepted: February 28, 2017

Published: March 10, 2017

Copyright: Shi et al. This is an open-access article distributed under the terms of the Creative Commons Attribution License (CC-BY), which permits unrestricted use, distribution, and reproduction in any medium, provided the original author and source are credited.

\section{ABSTRACT}

Background: The relationship between marital status and oral cavity squamous cell carcinoma (OCSCC) survival has not been explored. The objective of our study was to evaluate the impact of marital status on OCSCC survival and investigate the potential mechanisms.

Results: Married patients had better 5-year cancer-specific survival (CSS) $(66.7 \%$ vs $54.9 \%$ ) and 5 -year overall survival (OS) (56.0\% vs $41.1 \%)$. In multivariate Cox regression models, unmarried patients also showed higher mortality risk for both CSS (Hazard Ratio [HR]: 1.260, 95\% confidence interval (CI): 1.187-1.339, $P<0.001$ ) and OS (HR: 1.328, 95\% CI: 1.266-1.392, $P<0.001$ ). Multivariate logistic regression showed married patients were more likely to be diagnosed at earlier stage $(P<0.001)$ and receive surgery $(P<\mathbf{0 . 0 0 1})$. Married patients still demonstrated better prognosis in the $1: 1$ matched group analysis (CSS: $62.9 \%$ vs $60.8 \%$, OS: $52.3 \%$ vs $46.5 \%$ ).

Materials and Methods: 11022 eligible OCSCC patients were identified from Surveillance, Epidemiology, and End Results (SEER) database, including 5902 married and 5120 unmarried individuals. Kaplan-Meier analysis, Log-rank test and Cox proportional hazards regression model were used to analyze survival and mortality risk. Influence of marital status on stage, age at diagnosis and selection of treatment was determined by binomial and multinomial logistic regression. Propensity score matching method was adopted to perform a 1:1 matched cohort.

Conclusions: Marriage has an independently protective effect on OCSCC survival. Earlier diagnosis and more sufficient treatment are possible explanations. Besides, even after 1:1 matching, survival advantage of married group still exists, indicating that spousal support from other aspects may also play an important role.

\section{INTRODUCTION}

Approximately over 200,000 new cases are diagnosed with oral cavity cancer (OCC) annually in the world and OCC leads to more than 100,000 deaths globally [1]. Oral cavity squamous cell carcinoma (OCSCC) accounts for the vast majority of total OCC and is generally considered to be closely related with tobacco and alcohol use [2]. In the United States, the incidence of OCSCC decreases slightly in recent years $[3,4]$, however in Australia and many regions of Western Europe, the morbidity continues to increase $[5,6]$. It's also noteworthy that in developing countries, OCSCC is still one of the ten leading causes for cancer death in male population [1].

It is a common understanding that social support, especially spousal support plays an important role in improving cancer outcomes. Many researches in recent years have demonstrated that marital status could independently affect survival in several cancer types [7-11]. Similarly, Inverso G et al. reported married patients with head and neck cancer had a longer survival than those unmarried, however in that study, researchers only took cancer-specific survival (CSS) into consideration while neglected overall survival (OS), which might benefit more from spousal support. Additionally, they were not able 
to discuss if marriage would affect survival from other aspects besides of favoring early diagnosis and adequate treatment [12]. Therefore, to the best of our knowledge, there is a dearth of researches focusing on the significance of marital status on OCSCC survival and elucidating relatively comprehensive mechanisms. In this study, we used data from Surveillance, Epidemiology, and End Results (SEER) database to investigate the influence of marital status on OCSCC survival and propose potential explanations in detail.

\section{RESULTS}

\section{Patient baseline characteristics}

Totally, we identified 11022 eligible OCSCC patients diagnosed between 1988 and 2008 in our study. All the patients in our study were actively followed up. Of these 11022 cases, 5902 (53.5\%) were married and $5120(46.5 \%)$ were unmarried. $2.7 \%$ of unmarried patients came up with distant metastasis while the metastatic rate in the married group was $1.5 \%$. Demographics and clinicopathological characteristics of enrolled patients are listed in Table 1. Significant differences $(P<0.001)$ were observed in all variables by Pearson Chi-squared $\left(\chi^{2}\right)$ test except for year $(P=0.098)$ and grade $(P=0.138)$. For better analysis, we listed stage IVc which stands for distant metastasis (M1) apart from Stage IVa/IVb because of different prognosis. We observed that patients in the married group were more likely to be white and male and had a higher proportion of tongue cancer. Besides, in general, married group also displayed earlier stage at diagnosis and a higher rate of receiving surgery, however, unmarried patients seemed more likely to be treated with radiotherapy than those married (Table 1).

\section{Impact of marital status on cancer-specific survival of OCSCC patients}

We used Kaplan-Meier analysis and Log-rank test to evaluate the impact of marital status on CSS of OCSCC patients (Figure 1A). In summary, the married group had a better 5-year CSS (66.7\% vs 54.9\%) than those unmarried. These prognostic differences were also significant in the univariate Log-rank test $(P<0.001)$. In the univariate analysis, gender $(P=0.013)$, age $(P<0.001)$, site $(P<0.001)$, race $(P<0.001)$, grade $(P<0.001)$, TNM stage $(P<0.001)$, surgery $(P<0.001)$ and radiotherapy $(P<0.001)$ were also significantly associated with cancerspecific survival of OCSCC patients and these variables were all included in the following multivariate Cox analysis (Table 2).

In the Cox regression, we found that unmarried group had a significantly increasing risk for cancerspecific mortality (HR 1.260, 95\%CI 1.187-1.339, $P<0.001)$. Besides, age, gender, primary site, grade, race, TNM stage at diagnosis, surgery and radiotherapy were validated as independent risk or protective factors as well. It is noteworthy that contradictory to common sense, we observed a better 5-year CSS in the no-radiotherapy group $(68.3 \%)$ than those who received radiotherapy (RT) $(58.2 \%)$, complicated influence of unadjusted confounders was a possible reason and receiving RT still demonstrated a protective effect on cancer-specific survival (compared with no-RT group, HR: 0.716 , 95\% CI 0.669-0.767, $P<0.001$ ) after multivariate Cox regression (Table 2).

\section{Impact of marital status on overall survival of OCSCC patients}

Subsequently, we also assessed the impact of marital status on OS of OCSCC patients. (Figure 1B) Married group had a better 5-year OS (55.9\% vs $41.1 \%)$ than unmarried patients and the difference was significant in Log-rank test $(P<0.001)$. Furthermore in the univariate analysis, all the baseline characteristics including gender $(P=0.011)$, age $(P<0.001)$, site $(P<0.001)$, race $(P<0.001)$, year $(P=0.002)$, grade $(P<0.001)$, TNM stage $(P<0.001)$, surgery $(P<0.001)$ and radiotherapy $(P<0.001)$ were also correlated with overall survival and they were further adjusted in multivariate Cox regression (Table 3).

In the multivariate analysis, unmarried status significantly increased overall mortality risk (HR 1.260, 95\% CI 1.187-1.339, $P<0.001)$. Besides, other covariates including age, gender, primary site, grade, race, stage at diagnosis, surgery, and radiotherapy also proved to be independent prognostic factors for overall survival. The results are summarized in Table 3.

\section{Effect of marital status on TNM stage at diagnosis}

From the baseline characteristics in Table 1, we noticed that married patients had a higher prevalence of stage I/II (57.0\%) than unmarried patients $(47.5 \%)$. We reasonably hypothesized that one important reason for the survival advantage of married group was early diagnosis. If the hypothesis was true, after multivariate adjustment, unmarried individuals should still take higher risk of being diagnosed at a more advanced stage. Relevance between marital status and stage at diagnosis was displayed by univariate and multivariate binomial logistic regression models (Table 4). Race, gender, primary site, grade, age, surgery and radiotherapy were all validated to be related with TNM stage at diagnosis in the univariate logistic regression, and then these variables were adjusted in the multivariate logistic analysis. The result showed unmarried patients were significantly more likely to be diagnosed at stage III or stage IV (compared with married patients, OR: 1.288, 95\% CI: 1.182-1.402, $P<0.001)$. Consequently, there was clear evidence that marriage benefited OCSCC prognosis through earlier diagnosis. 
Table 1: Baseline characteristics of OCSCC patients by marital status

\begin{tabular}{|c|c|c|c|c|}
\hline \multirow{2}{*}{ Characteristic } & Total & Married & Unmarried & \multirow{2}{*}{$P$} \\
\hline & $11022(100)$ & $5902(100)$ & $5120(100)$ & \\
\hline Gender & & & & $<0.001$ \\
\hline Female & $4163(37.8)$ & $1909(32.3)$ & $2254(44.0)$ & \\
\hline Male & $6859(62.2)$ & $3993(67.7)$ & $2866(56.0)$ & \\
\hline Age & & & & $<0.001$ \\
\hline$<35$ & $226(2.1)$ & $107(1.8)$ & $119(2.3)$ & \\
\hline $35-44$ & $764(6.9)$ & $412(7.0)$ & $352(6.9)$ & \\
\hline $45-54$ & $2164(19.6)$ & $1156(19.6)$ & $1008(19.7)$ & \\
\hline $55-64$ & $2909(26.4)$ & 1647 (27.9) & $1262(24.6)$ & \\
\hline $65-74$ & $2587(23.5)$ & $1532(26.0)$ & $1055(20.6)$ & \\
\hline $75-84$ & 1785 (16.2) & $860(14.6)$ & $925(18.1)$ & \\
\hline$>85$ & $587(5.3)$ & $188(3.2)$ & $399(7.8)$ & \\
\hline ICD-O-3 site code ${ }^{1}$ & & & & $<0.001$ \\
\hline Tongue $^{2}$ & $3963(36.0)$ & $2293(38.9)$ & $1670(32.6)$ & \\
\hline Gum and other mouth & $3846(34.9)$ & $2035(34.5)$ & $1811(35.4)$ & \\
\hline Floor of mouth & $3213(29.2)$ & $1574(26.7)$ & $1639(32.0)$ & \\
\hline Race & & & & $<0.001$ \\
\hline White & $9041(82.0)$ & $4944(83.8)$ & $4097(80.0)$ & \\
\hline Black & $1089(9.9)$ & $351(5.9)$ & $738(14.4)$ & \\
\hline Other $^{3}$ & $856(7.8)$ & $589(10.0)$ & $267(5.2)$ & \\
\hline Unknown & $36(0.3)$ & $18(0.3)$ & $18(0.4)$ & \\
\hline Grade & & & & 0.138 \\
\hline Well/Moderately differentiated & $8681(78.8)$ & $4679(79.3)$ & $4002(78.2)$ & \\
\hline Poorly/Undifferentiated & $1906(17.3)$ & $1011(17.1)$ & $895(17.5)$ & \\
\hline Unknown & $435(3.9)$ & $212(3.6)$ & $223(4.4)$ & \\
\hline Year & & & & 0.098 \\
\hline 1988-1994 & $1540(14.0)$ & $857(14.5)$ & $683(13.3)$ & \\
\hline 1995-2001 & $3172(28.8)$ & $1660(28.1)$ & $1512(29.5)$ & \\
\hline 2002-2008 & $6310(57.2)$ & $3385(57.4)$ & $2925(57.1)$ & \\
\hline TNM Stage & & & & $<0.001$ \\
\hline I & $3591(32.6)$ & $2200(37.3)$ & $1391(27.2)$ & \\
\hline II & $2201(20.0)$ & $1162(19.7)$ & $1039(20.3)$ & \\
\hline III & $1263(11.5)$ & $613(10.4)$ & $650(12.7)$ & \\
\hline $\mathrm{IVa} / \mathrm{IVb}$ & $3741(33.9)$ & $1837(31.1)$ & $1904(37.2)$ & \\
\hline IVc & $226(2.1)$ & $90(1.5)$ & $136(2.7)$ & \\
\hline Surgery & & & & $<0.001$ \\
\hline No surgery & $1972(17.9)$ & $797(13.5)$ & $1175(22.9)$ & \\
\hline Local excision/destruction ${ }^{4}$ & $1953(17.7)$ & $1131(19.2)$ & $822(16.1)$ & \\
\hline Wide/Radical excision & $7097(64.4)$ & $3974(67.3)$ & $3123(61.0)$ & \\
\hline Radiotherapy & & & & $<0.001$ \\
\hline No & $5490(49.8)$ & $3087(52.3)$ & $2403(46.9)$ & \\
\hline Yes & $5532(50.2)$ & $2815(47.7)$ & $2717(53.1)$ & \\
\hline
\end{tabular}

SEER 1988-2008 $(n=11022)$.

${ }^{1}$ Lip was not included because NCCN regards lip and oral cavity as two different parts and provides different treatment guidelines respectively.

${ }^{2}$ Subsites of the tongue regarded as anatomic part of oropharynx were not included.

${ }^{3}$ Includes American Indian/Alaska Native, Asian/Pacific Islander.

${ }^{4}$ Local destruction includes photodynamic therapy, electrocautery, cryosurgery, laser ablation, etc. 


\section{Relationship between marital status and age at diagnosis}

Age was another crucial prognostic factor. To investigate whether married patients were younger at diagnosis than those unmarried, we performed a multivariate multinomial logistic regression to explore the association between marital status and age at diagnosis. When defining the $<35$ age subgroup as base outcome for reference, we observed that compared with unmarried patients, married people were more likely to be 55-64 (OR: $1.717,95 \%$ CI: $1.137-2.593, P<0.001$ ), 65-74 (OR: $2.004,95 \%$ CI: $1.508-2.604, P<0.001)$ and $75-84$ (OR: $1.356,95 \%$ CI: $1.015-1.811, P=0.039$ ) years old at diagnosis. However, there was no significant difference between married and unmarried patients in 35-44 (OR: 1.398, 95\% CI: $0.667-2.930, P=0.374), 45-54$ (OR: $1.670,95 \%$ CI: $0.743-3.755, P=0.215)$ and $>85$ age subgroups (OR: $0.766,95 \%$ CI: $0.552-1.063, P=0.111$ ). Therefore, age might not be the main explanation for survival benefits of marriage (Table 5).

\section{Effect of marital status on treatment selection}

Adequate treatment could be another potential cause for longevity among married patients. To investigate whether marital status influenced survival by selection of treatment, we analyzed the preference for receiving surgery or radiotherapy by multivariate logistic regression (Table 5). In the multivariate logistic analysis for surgery, we found that married patients were more likely to undergo surgery, for both local destruction/excision (OR: 1.686, 95\% CI: $1.459-1.948, P<0.001)$ and total/radical surgery (OR: 1.606, 95\%CI: 1.404-1.800, $P<0.001$ ). Nevertheless in the multivariate logistic analysis for RT, significant preference of radiotherapy was not observed between married and unmarried groups (OR: 1.017, 95\% CI: $0.932-1.110, P=0.700$ ).

Therefore, in addition to earlier diagnosis, better prognosis of married OCSCC patients was also attributed to more sufficient treatment, especially surgical treatment.

\section{Subgroup analysis for evaluating the effect of marital status on OS and CSS}

We have certified that delayed diagnosis and undertreatment were important reasons for compromised survival of unmarried patients. Next we separately stratified stage at diagnosis, age and treatment condition, then analyzed survival in each subgroup to see if marriage still benefited patients' prognosis in subgroup multivariate Cox analysis.

Firstly, using multivariate Cox regression, we assessed the effect of marital status on CSS and OS at each stage, in patients with stage I to stage $I V a / I V b$ at diagnosis, marriage always played a significant protective role in both CSS and OS. For overall survival of stage IVc patients, marriage was also a probable beneficial factor though not significant at $95 \%$ confidence level (HR: 1.394, 95\% CI: $0.989-1.964, P=0.057)$. Of note, for all the five stage subgroups, the hazard ratios of unmarried patients for OS were always greater than those for CSS, indicating marital status had a stronger impact on overall survival than cancer-specific survival (Table 6) (Figure 2).

Secondly, we conducted multivariate Cox regression adjusting for the aforementioned variables and assessed
A

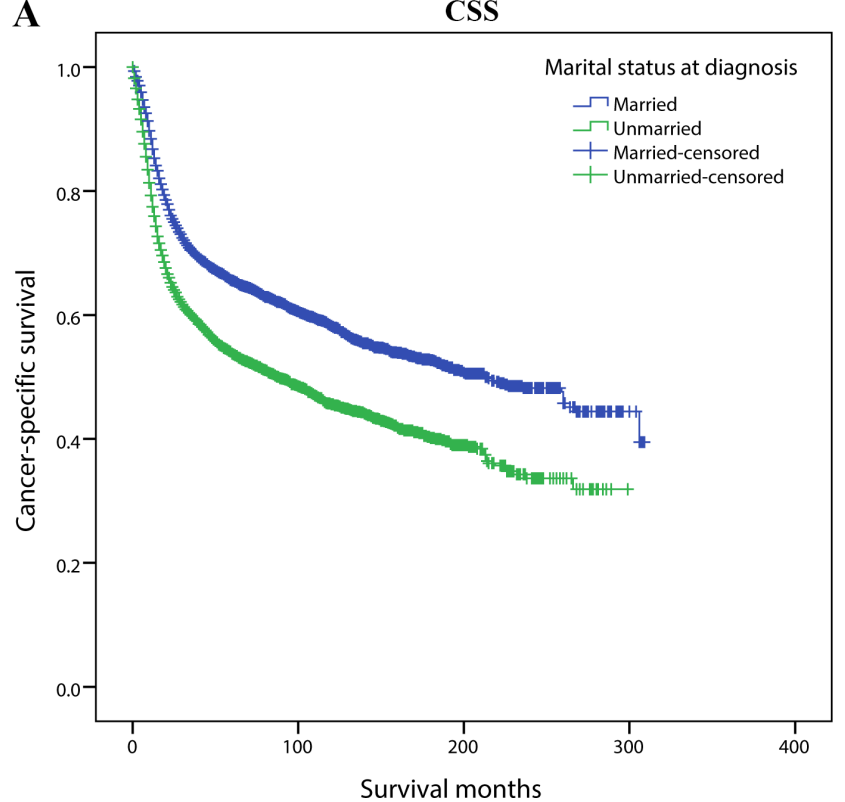

B $\quad$ os

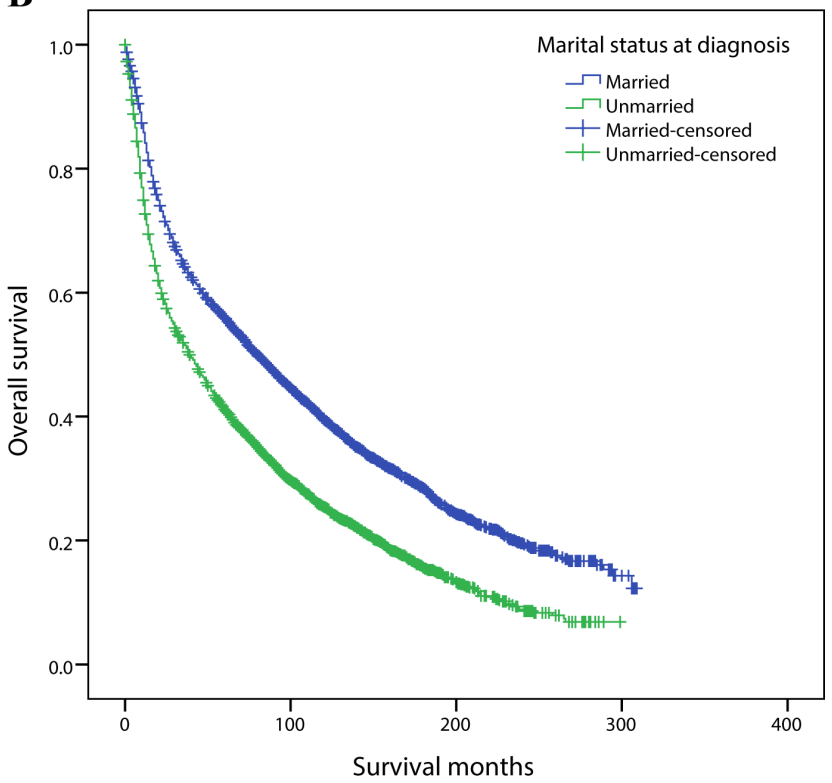

Figure 1: Kaplan-Meier survival curves:cancer-specific survival and overall survival in 11022 OCSCC patients. Married vs Unmarried (A) Cancer-specific survival: $\chi^{2}=235.220, P<0.001$; (B) Overall survival: $\chi^{2}=329.475, P<0.001$. 
Table 2: Univariate and multivariate analysis for evaluating the CSS predictors of OCSCC patients

\begin{tabular}{|c|c|c|c|c|c|c|}
\hline \multirow{2}{*}{ Variable } & \multirow{2}{*}{ 5-year CSS } & \multicolumn{2}{|c|}{ Univariate Analysis } & \multicolumn{3}{|c|}{ Multivariate Analysis } \\
\hline & & Log-rank $\chi^{2}$ & $P$ & HR & $95 \%$ CI & $P$ \\
\hline Marital status & & 183.135 & $<0.001$ & & & \\
\hline Married & $66.7 \%$ & & & Reference & & \\
\hline Unmarried & $54.9 \%$ & & & 1.26 & $1.187-1.339$ & $<0.001$ \\
\hline Gender & & 6.189 & 0.013 & & & \\
\hline Female & $62.1 \%$ & & & Reference & & \\
\hline Male & $59.7 \%$ & & & 1.068 & $1.003-1.138$ & 0.041 \\
\hline Age & & 227.992 & $<0.001$ & & & \\
\hline$<35$ & $74.7 \%$ & & & 0.326 & $0.246-0.432$ & $<0.001$ \\
\hline $35-44$ & $66.9 \%$ & & & 0.47 & $0.396-0.558$ & $<0.001$ \\
\hline $45-54$ & $64.7 \%$ & & & 0.497 & $0.431-0.573$ & $<0.001$ \\
\hline $55-64$ & $62.9 \%$ & & & 0.543 & $0.474-0.622$ & $<0.001$ \\
\hline $65-74$ & $61.7 \%$ & & & 0.612 & $0.534-0.700$ & $<0.001$ \\
\hline $75-84$ & $53.8 \%$ & & & 0.815 & $0.710-0.934$ & 0.003 \\
\hline$>85$ & $45.6 \%$ & & & Reference & & \\
\hline ICD-O-3 site code & & 109.635 & $<0.001$ & & & \\
\hline Tongue & $66.7 \%$ & & & Reference & & \\
\hline Gum and other mouth & $57.6 \%$ & & & 0.964 & $0.896-1.037$ & 0.326 \\
\hline Floor of mouth & $58.9 \%$ & & & 1.096 & $1.016-1.182$ & 0.017 \\
\hline Race & & 139.129 & $<0.001$ & & & \\
\hline White & $63.2 \%$ & & & Reference & & \\
\hline Black & $46.8 \%$ & & & 1.228 & $1.123-1.343$ & $<0.001$ \\
\hline Other & $58.9 \%$ & & & 1.134 & $1.018-1.263$ & 0.022 \\
\hline Unknown & $82.7 \%$ & & & 0.252 & $0.081-0.781$ & 0.017 \\
\hline Grade & & 190.713 & $<0.001$ & & & \\
\hline Well/Moderately differentiated & $64.3 \%$ & & & Reference & & \\
\hline Poorly/Undifferentiated & $47.6 \%$ & & & 1.351 & $1.259-1.451$ & $<0.001$ \\
\hline Unknown & $60.5 \%$ & & & 0.945 & $0.807-1.106$ & 0.481 \\
\hline Year & & 2.019 & 0.364 & & & NI \\
\hline 1988-1994 & $60.5 \%$ & & & & & \\
\hline 1995-2001 & $61.9 \%$ & & & & & \\
\hline 2002-2008 & $61.2 \%$ & & & & & \\
\hline TNM Stage & & 1529.025 & $<0.001$ & & & \\
\hline I & $81.5 \%$ & & & Reference & & \\
\hline II & $65.8 \%$ & & & 1.477 & $1.339-1.629$ & $<0.001$ \\
\hline III & $52.8 \%$ & & & 1.974 & $1.769-2.204$ & $<0.001$ \\
\hline $\mathrm{IVa} / \mathrm{IVb}$ & $43.7 \%$ & & & 2.583 & $2.370-2.814$ & $<0.001$ \\
\hline IVc & $18.2 \%$ & & & 4.871 & $4.111-5.771$ & $<0.001$ \\
\hline Surgery & & 1392.293 & $<0.001$ & & & \\
\hline No surgery & $30.2 \%$ & & & Reference & & \\
\hline Local excision/destruction & $74.7 \%$ & & & 0.458 & $0.413-0.509$ & $<0.001$ \\
\hline Wide/Radical excision & $65.5 \%$ & & & 0.499 & $0.464-0.536$ & $<0.001$ \\
\hline Radiotherapy & & 557.125 & $<0.001$ & & & \\
\hline No & $68.3 \%$ & & & Reference & & \\
\hline Yes & $58.2 \%$ & & & 0.716 & $0.669-0.767$ & $<0.001$ \\
\hline
\end{tabular}

SEER 1988-2008 $(n=11022)$.

Abbreviations: HR: Hazard ratio; CI: confidence interval; NI: Not Included. 
Table 3: Univariate and multivariate analysis for evaluating the OS predictors of OCSCC patients

\begin{tabular}{|c|c|c|c|c|c|c|}
\hline \multirow{2}{*}{ Variable } & \multirow{2}{*}{ 5-year OS } & \multicolumn{2}{|c|}{ Univariate Analysis } & \multicolumn{3}{|c|}{ Multivariate Analysis } \\
\hline & & Log-rank $\chi^{2}$ & $\boldsymbol{P}$ & HR & $95 \% \mathrm{CI}$ & $\boldsymbol{P}$ \\
\hline Marital status & & 329.475 & $<0.001$ & & & \\
\hline Married & $55.9 \%$ & & & Reference & & \\
\hline Unmarried & $41.1 \%$ & & & 1.328 & $1.266-1.392$ & $<0.001$ \\
\hline Gender & & 6.47 & 0.011 & & & \\
\hline Female & $50.7 \%$ & & & Reference & & \\
\hline Male & $48.1 \%$ & & & 1.201 & $1.143-1.262$ & $<0.001$ \\
\hline Age & & 1195.931 & $<0.001$ & & & \\
\hline$<35$ & $73.5 \%$ & & & 0.136 & $0.105-0.176$ & $<0.001$ \\
\hline $35-44$ & $62.8 \%$ & & & 0.232 & $0.202-0.266$ & $<0.001$ \\
\hline $45-54$ & $56.8 \%$ & & & 0.281 & $0.253-0.313$ & $<0.001$ \\
\hline $55-64$ & $52.4 \%$ & & & 0.355 & $0.321-0.393$ & $<0.001$ \\
\hline $65-74$ & $48.1 \%$ & & & 0.457 & $0.414-0.505$ & $<0.001$ \\
\hline $75-84$ & $36.2 \%$ & & & 0.694 & $0.629-0.766$ & $<0.001$ \\
\hline$>85$ & $20.8 \%$ & & & Reference & & \\
\hline ICD-O-3 site code & & 263.598 & $<0.001$ & & & \\
\hline Tongue & $56.8 \%$ & & & Reference & & \\
\hline Gum and other mouth & $44.8 \%$ & & & 1.01 & $0.954-1.070$ & 0.73 \\
\hline Floor of mouth & $44.7 \%$ & & & 1.231 & $1.160-1.306$ & $<0.001$ \\
\hline Race & & 130.039 & $<0.001$ & & & \\
\hline White & $50.5 \%$ & & & Reference & & \\
\hline Black & $34.8 \%$ & & & 1.191 & $1.107-1.282$ & $<0.001$ \\
\hline Other & $51.2 \%$ & & & 0.947 & $0.866-1.037$ & 0.242 \\
\hline Unknown & $77.2 \%$ & & & 0.511 & $0.283-0.924$ & 0.026 \\
\hline Grade & & 180.103 & $<0.001$ & & & \\
\hline Well/Moderately differentiated & $51.8 \%$ & & & Reference & & \\
\hline Poorly/Undifferentiated & $36.8 \%$ & & & 1.284 & $1.212-1.360$ & $<0.001$ \\
\hline Unknown & $49.0 \%$ & & & 0.963 & $0.845-1.097$ & 0.568 \\
\hline Year & & 12.226 & 0.002 & & & \\
\hline 1988-1994 & $45.9 \%$ & & & Reference & & \\
\hline $1995-2001$ & $49.0 \%$ & & & 0.906 & $0.846-0.970$ & $<0.005$ \\
\hline 2002-2008 & $49.9 \%$ & & & 0.927 & $0.867-0.992$ & 0.027 \\
\hline TNM Stage & & 1277.985 & $<0.001$ & & & \\
\hline I & $68.8 \%$ & & & Reference & & \\
\hline II & $52.2 \%$ & & & 1.25 & $1.165-1.341$ & $<0.001$ \\
\hline III & $39.8 \%$ & & & 1.598 & $1.470-1.737$ & $<0.001$ \\
\hline $\mathrm{IVa} / \mathrm{IVb}$ & $33.6 \%$ & & & 1.88 & $1.766-2.000$ & $<0.001$ \\
\hline IVc & $13.6 \%$ & & & 3.101 & $2.672-3.598$ & $<0.001$ \\
\hline Surgery & & 1609.765 & $<0.001$ & & & \\
\hline No surgery & $19.0 \%$ & & & Reference & & \\
\hline Local excision/destruction & $61.5 \%$ & & & 0.512 & $0.472-0.554$ & $<0.001$ \\
\hline Wide/Radical excision & $54.0 \%$ & & & 0.528 & $0.497-0.561$ & $<0.001$ \\
\hline Radiotherapy & & 657.205 & $<0.001$ & & & \\
\hline No & $59.1 \%$ & & & Reference & & \\
\hline Yes & $47.1 \%$ & & & 0.787 & $0.746-0.829$ & $<0.001$ \\
\hline
\end{tabular}

SEER 1988-2008 $(n=11022)$. 
Table 4: Characteristics of patients by TNM stage at diagnosis using univariate and multivariate binary logistic regression

\begin{tabular}{|c|c|c|c|c|c|c|}
\hline \multirow{2}{*}{ Characteristics } & \multirow{2}{*}{$\begin{array}{c}\text { Stage I/II } \\
5792(100)\end{array}$} & \multirow{2}{*}{$\begin{array}{c}\text { Stage III/IV } \\
5230(100)\end{array}$} & \multirow{2}{*}{$\begin{array}{c}\text { Univariate } \\
\text { Analysis }\end{array}$} & \multicolumn{3}{|c|}{ Multivariate Analysis } \\
\hline & & & & Odds Ratio & $95 \% \mathrm{CI}$ & $\boldsymbol{P}$ \\
\hline Marital status & & & $<0.001$ & & & \\
\hline Married & $3362(58.0)$ & $2540(48.6)$ & & Reference & & \\
\hline Unmarried & $2430(42.0)$ & $2690(51.4)$ & & 1.288 & $1.182-1.402$ & $<0.001$ \\
\hline Gender & & & $<0.001$ & & & \\
\hline Female & $2290(39.5)$ & $1873(35.8)$ & & Reference & & \\
\hline Male & $3502(60.5)$ & $3357(64.2)$ & & 1.094 & $1.001-1.196$ & 0.047 \\
\hline Age & & & 0.006 & & & \\
\hline$<35$ & $131(2.3)$ & $95(1.8)$ & & 0.886 & $0.629-1.248$ & 0.488 \\
\hline $35-44$ & $407(7.0)$ & $357(6.8)$ & & 1.005 & $0.786-1.284$ & 0.969 \\
\hline $45-54$ & 1104 (19.1) & $1060(20.3)$ & & 0.969 & $0.786-1.195$ & 0.767 \\
\hline $55-64$ & $1480(25.6)$ & $1429(27.3)$ & & 0.93 & $0.759-1.140$ & 0.485 \\
\hline $65-74$ & $1414(24.4)$ & $1173(22.4)$ & & 0.83 & $0.678-1.017$ & 0.073 \\
\hline $75-84$ & $969(16.7)$ & $816(15.6)$ & & 0.816 & $0.663-1.005$ & 0.056 \\
\hline$>85$ & $287(5.0)$ & $300(5.7)$ & & Reference & & \\
\hline ICD-O-3 site code & & & $<0.001$ & & & \\
\hline Tongue & $2442(42.2)$ & $1521(29.1)$ & & Reference & & \\
\hline Gum and other mouth & $1763(30.4)$ & $2083(39.8)$ & & 1.648 & $1.489-1.824$ & $<0.001$ \\
\hline Floor of mouth & $1587(27.4)$ & $1626(31.1)$ & & 1.432 & $1.288-1.593$ & $<0.001$ \\
\hline Race & & & $<0.001$ & & & \\
\hline White & $4904(84.7)$ & $4137(79.1)$ & & Reference & & \\
\hline Black & $398(6.9))$ & $691(13.2)$ & & 1.521 & $1.317-1.756$ & $<0.001$ \\
\hline Other & $464(8.0)$ & $392(7.5)$ & & 1.094 & $0.937-1.278$ & 0.256 \\
\hline Unknown & $26(0.4)$ & $10(0.2)$ & & 0.66 & $0.305-1.426$ & 0.290 \\
\hline Grade & & & $<0.001$ & & & \\
\hline Well/Moderately differentiated & $4765(82.3)$ & $3916(74.9)$ & & Reference & & \\
\hline Poorly/Undifferentiated & $778(13.4)$ & $1128(21.6)$ & & 1.502 & $1.346-1.675$ & $<0.001$ \\
\hline Unknown & $249(4.3)$ & $186(3.6)$ & & 0.717 & $0.573-0.896$ & 0.004 \\
\hline Year & & & 0.487 & & & NI \\
\hline 1988-1994 & $788(13.6)$ & $752(14.4)$ & & & & \\
\hline 1995-2001 & $1680(29.0)$ & $1492(28.5)$ & & & & \\
\hline $2002-2008$ & $3324(57.4)$ & $2986(57.1)$ & & & & \\
\hline Surgery & & & $<0.001$ & & & \\
\hline No surgery & $561(9.7)$ & $1411(27.0)$ & & Reference & & \\
\hline Local excision/destruction & $1404(24.2)$ & $549(10.5)$ & & 0.281 & $0.242-0.327$ & $<0.001$ \\
\hline Wide/Radical excision & $3827(66.1)$ & $3270(62.5)$ & & 0.499 & $0.443-0.562$ & $<0.001$ \\
\hline Radiotherapy & & & $<0.001$ & & & \\
\hline No & $3856(66.6)$ & $1634(31.2)$ & & Reference & & \\
\hline Yes & $1936(33.4)$ & $3596(68.8)$ & & 3.383 & $3.108-3.681$ & $<0.001$ \\
\hline
\end{tabular}

SEER 1988-2008 $(n=11022)$.

Abbreviations: CI: confidence interval; NI: Not Included. 
Table 5: Multinomial multivariate logistic analysis of age at diagnosis and treatment according to marital status

\begin{tabular}{|c|c|c|c|}
\hline \multirow{2}{*}{ Variable } & \multicolumn{3}{|c|}{ Multivariate multinomial logistic analysis } \\
\hline & Odds Ratio & $95 \% \mathrm{CI}$ & $\boldsymbol{P}$ \\
\hline \multicolumn{4}{|c|}{ Age at diagnosis } \\
\hline \multicolumn{4}{|c|}{$<35$} \\
\hline Unmarried & As base outcome & & \\
\hline \multicolumn{4}{|l|}{ Married } \\
\hline \multicolumn{4}{|l|}{$35-44$} \\
\hline Unmarried & Reference & & \\
\hline Married & 1.398 & $0.667-2.930$ & 0.374 \\
\hline \multicolumn{4}{|l|}{$45-54$} \\
\hline Unmarried & Reference & & \\
\hline Married & 1.670 & $0.743-3.755$ & 0.215 \\
\hline \multicolumn{4}{|l|}{$55-64$} \\
\hline Unmarried & Reference & & \\
\hline Married & 1.717 & $1.137-2.593$ & 0.010 \\
\hline \multicolumn{4}{|l|}{$65-74$} \\
\hline Unmarried & Reference & & \\
\hline Married & 2.004 & $1.508-2.604$ & $<0.001$ \\
\hline \multicolumn{4}{|l|}{$75-84$} \\
\hline Unmarried & Reference & & \\
\hline Married & 1.356 & $1.015-1.811$ & 0.039 \\
\hline \multicolumn{4}{|l|}{$>85$} \\
\hline Unmarried & Reference & & \\
\hline Married & 0.766 & $0.552-1.063$ & 0.111 \\
\hline \multicolumn{4}{|l|}{ Surgery } \\
\hline \multicolumn{4}{|l|}{ No surgery } \\
\hline Unmarried & As base outcome & & \\
\hline \multicolumn{4}{|l|}{ Married } \\
\hline \multicolumn{4}{|c|}{ Local excision/destruction } \\
\hline Unmarried & Reference & & \\
\hline Married & 1.686 & $1.459-1.948$ & $<0.001$ \\
\hline \multicolumn{4}{|c|}{ Wide/Radical excision } \\
\hline Unmarried & Reference & & \\
\hline Married & 1.606 & $1.404-1.800$ & $<0.001$ \\
\hline \multicolumn{4}{|l|}{ Radiotherapy } \\
\hline \multicolumn{4}{|c|}{ No radiotherapy } \\
\hline Unmarried & As base outcome & & \\
\hline \multicolumn{4}{|l|}{ Married } \\
\hline \multicolumn{4}{|c|}{ Receive radiotherapy } \\
\hline Unmarried & Reference & & \\
\hline Married & 1.017 & $0.932-1.110$ & 0.700 \\
\hline
\end{tabular}

SEER 1988-2008 $(n=11022)$. 
Table 6: Multivariate subgroup analysis of marital status on OCSCC overall and cancer-specific survival according to TNM stage, age at diagnosis and treatment

\begin{tabular}{|c|c|c|c|c|c|c|}
\hline \multirow{2}{*}{ Variable } & \multicolumn{3}{|c|}{ Multivariate Analysis (CSS) } & \multicolumn{3}{|c|}{ Multivariate Analysis (OS) } \\
\hline & HR & $95 \% \mathrm{CI}$ & $\boldsymbol{P}$ & HR & $95 \% \mathrm{CI}$ & $\boldsymbol{P}$ \\
\hline \multicolumn{7}{|l|}{ TNM Stage } \\
\hline \multicolumn{7}{|l|}{ Stage I } \\
\hline Married & Reference & & & Reference & & \\
\hline Unmarried & 1.132 & $0.984-1.303$ & 0.084 & 1.216 & $1.106-1.337$ & $<0.001$ \\
\hline \multicolumn{7}{|l|}{ Stage II } \\
\hline Married & Reference & & & Reference & & \\
\hline Unmarried & 1.339 & $1.163-1.541$ & $<0.001$ & 1.37 & $1.232-1.524$ & $<0.001$ \\
\hline \multicolumn{7}{|l|}{ Stage III } \\
\hline Married & Reference & & & Reference & & \\
\hline Unmarried & 1.205 & $1.015-1.430$ & 0.033 & 1.402 & $1.219-1.613$ & $<0.001$ \\
\hline \multicolumn{7}{|c|}{ Stage IVa/IVb } \\
\hline Married & Reference & & & Reference & & \\
\hline Unmarried & 1.277 & $1.169-1.396$ & $<0.001$ & 1.309 & $1.215-1.411$ & $<0.001$ \\
\hline \multicolumn{7}{|l|}{ Stage IVc } \\
\hline Married & Reference & & & Reference & & \\
\hline Unmarried & 1.246 & $0.860-1.804$ & 0.245 & 1.394 & $0.989-1.964$ & 0.057 \\
\hline \multicolumn{7}{|c|}{ Age at diagnosis } \\
\hline \multicolumn{7}{|c|}{$<35$} \\
\hline Married & Reference & & & Reference & & \\
\hline \multirow{2}{*}{\multicolumn{7}{|c|}{$35-44$}} \\
\hline & & & & & & \\
\hline Married & Reference & & & Reference & & \\
\hline Unmarried & 1.254 & $0.972-1.619$ & 0.082 & 1.459 & $1.165-1.827$ & 0.001 \\
\hline \multicolumn{7}{|l|}{$45-54$} \\
\hline Married & Reference & & & Reference & & \\
\hline Unmarried & 1.335 & $1.158-1.540$ & $<0.001$ & 1.487 & $1.319-1.676$ & $<0.001$ \\
\hline \multicolumn{7}{|l|}{$55-64$} \\
\hline Married & Reference & & & Reference & & \\
\hline Unmarried & 1.299 & $1.155-1.461$ & $<0.001$ & 1.373 & $1.250-1.507$ & $<0.001$ \\
\hline \multicolumn{7}{|l|}{$65-74$} \\
\hline Married & Reference & & & Reference & & \\
\hline & 1.179 & $1.042-1.336$ & 0.009 & 1.23 & $1.120-1.350$ & $<0.001$ \\
\hline \multicolumn{7}{|l|}{$75-84$} \\
\hline Married & Reference & & & Reference & & \\
\hline Unmarried & 1.227 & $1.056-1.424$ & 0.007 & 1.237 & $1.108-1.381$ & $<0.001$ \\
\hline \multicolumn{7}{|l|}{$>85$} \\
\hline Married & Reference & & & Reference & & \\
\hline Unmarried & 1.106 & $0.826-1.480$ & 0.498 & 1.044 & $0.849-1.285$ & 0.682 \\
\hline \multicolumn{7}{|l|}{ Treatment } \\
\hline \multicolumn{7}{|c|}{ No surgery or radiotherapy } \\
\hline Married & Reference & & & Reference & & \\
\hline Unmarried & 1.328 & $1.014-1.738$ & 0.039 & 1.234 & $0.979-1.555$ & 0.076 \\
\hline Surgery onl & & & & & & \\
\hline Married & Reference & & & Reference & & \\
\hline Unmarried & 1.251 & $1.119-1.399$ & $<0.001$ & 1.331 & $1.232-1.438$ & $<0.001$ \\
\hline Radiotherap & & & & & & \\
\hline Married & Reference & & & Reference & & \\
\hline Unmarried & 1.207 & $1.056-1.381$ & 0.006 & 1.24 & $1.108-1.388$ & $<0.001$ \\
\hline Both surger & & & & & & \\
\hline Married & Reference & & & Reference & & \\
\hline Unmarried & 1.227 & $1.120-1.344$ & $<0.001$ & 1.287 & $1.193-1.384$ & $<0.001$ \\
\hline
\end{tabular}

SEER 1988-2008 $(n=11022)$. 

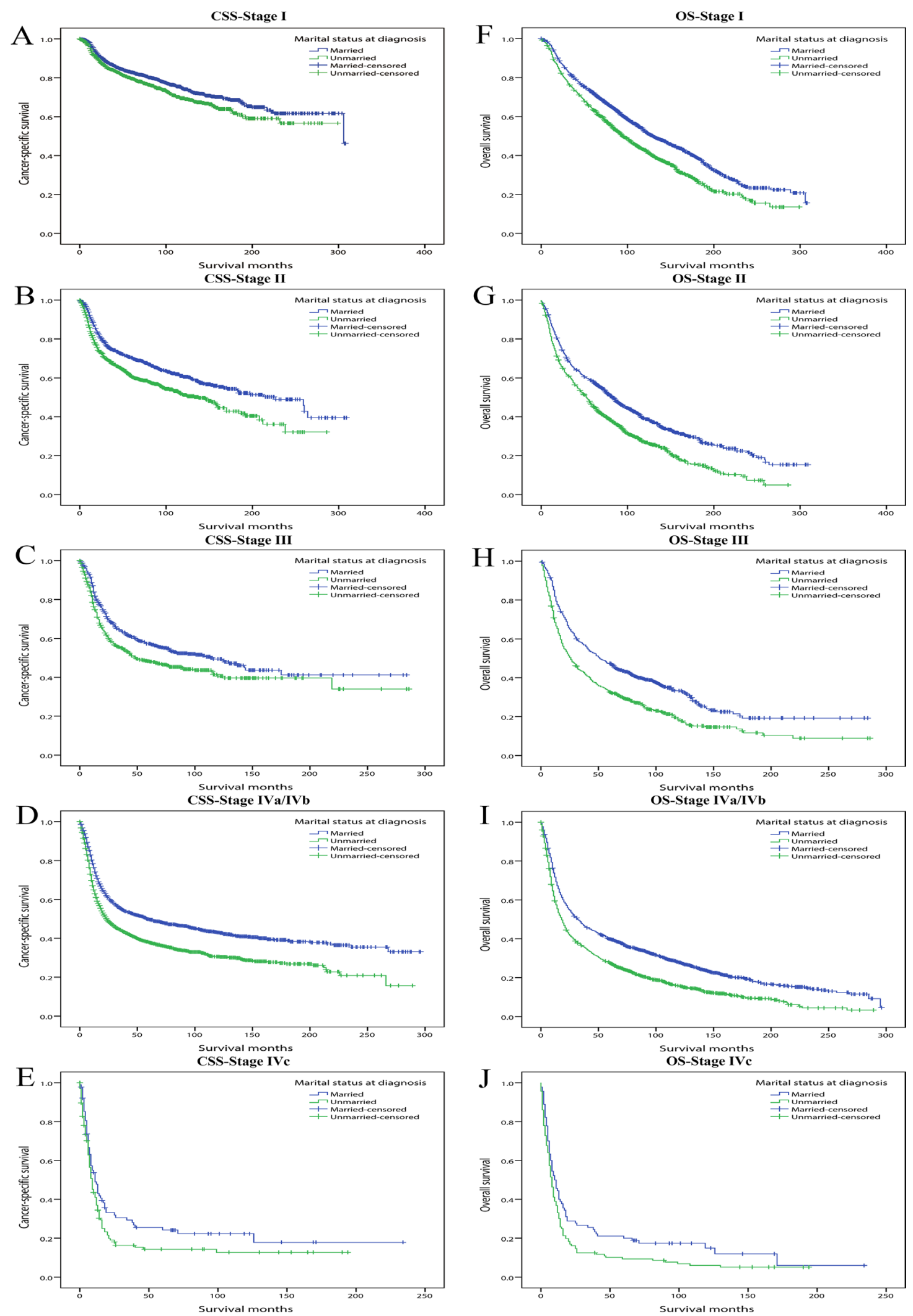

Figure 2: Kaplan-Meier survival curves: cancer-specific survival and overall survival in 11022 OCSCC patients according to TNM stage at diagnosis. (A) CSS-Stage I: $\chi^{2}=8.981, P=0.003$; (B) CSS-Stage II: $\chi^{2}=22.635, P<0.001$; (C) CSSStage III: $\chi^{2}=10.964, P<0.001$; (D) CSS-Stage IVa/IVb: $\chi^{2}=69.246, P<0.001$; (E) CSS-Stage IVc: $\chi^{2}=3.581, P=0.058$; (F) OS-Stage I: $\chi^{2}=43.523, P<0.001$; (G) OS-Stage II: $\chi^{2}=49.323, P<0.001$; (H) OS-Stage III: $\chi^{2}=39.349, P<0.001 ;$ (I) OS-Stage IVa/IVb: $\chi^{2}=101.648$, $P<0.001 ;(\mathbf{J})$ OS-Stage IVc: $\chi^{2}=5.398, P=0.020$. 
the influence of marital status on survival in each age subgroup. We observed that except for patients younger than 35 or older than 85 years old, unmarried status always significantly increased the mortality risk for both CSS and OS compared with married ones. Similar to stage subgroup analysis, for all the five age subgroups (35-44, 45-54, 55-64, 65-74, 75-84) in which marital status significantly influenced survival, marital status affected OS more greatly than CSS (Table 6). For concise illustration, Kaplan curves for CSS and OS were conducted based on binary age ( $\leq 60$ and $>60)$ subgroups (Figure 3 ).

Thirdly we divided patients into four subgroups which were No surgery or radiotherapy, Surgery only, Radiotherapy only and Both surgery and radiotherapy subgroups. In the multivariate Cox analysis, marriage always demonstrated protective role and marital status demonstrated stronger influence on OS than CSS (Table 6) (Figure 4).

In addition, we conducted subgroup analyses stratified by other baseline characteristics including gender, grade, primary site, year of diagnosis and race. The results are briefly summarized in two forest plots (CSS: Figure 5A; OS: Figure 5B). It was found that for both CSS and OS, compared with female, male patients displayed higher mortality risk when they were unmarried, illustrating marriage and its related spousal support might be more effective in men than women (CSS: HR for male unmarried group: 1.269, HR for female unmarried group: 1.225; OS: HR for male unmarried group: 1.347, HR for female unmarried group: 1.267). The impact of marital status was also stronger in white population than other ethnicity, gum squamous cell carcinoma patients than any other primary site, well/moderately differentiated tumor grade than those poorly differentiated or anaplastic.

\section{Survival analysis in 1:1 matched group}

As we've discussed above, married patients favored earlier diagnosis and more adequate treatment thus to benefit survival. Nevertheless, besides of these reasons, spousal support may also improve OCSCC prognosis from other aspects. In order to minimize the disturbance of confounding factors and ensure these confounders were not responsible for the outcomes, we utilized propensity score matching (PSM) method to perform a 1:1 matched cohort. Particularly, exact matching function was used for age, grade, TNM stage, surgery and radiation to make sure married and unmarried groups were identical in these variables. After matching, we obtained 6208 patients including 3104 married and another 3104 unmarried ones. Demographics and clinicopathological characteristics of the matched cohort are presented in Table 7. Besides of the exactly matched covariates which were totally the same $(P=1)$, other factors including gender $(P=0.854)$, site $(P=0.976)$, year $(P=0.295)$, race $(P=0.893)$ showed no significant difference as well.

Even so, in the survival analysis, married patients still demonstrated better prognosis than unmarried ones.
The 5-year CSS rate of married patients was $65.4 \%$ while the proportion of 5-year CSS was $60.6 \%$ in unmarried group. Similarly, the 5-year OS rate was $54.1 \%$ in the married group and $47.4 \%$ in unmarried group (Figure 6). In order to enhance persuasion, we also conducted multivariate Cox regression although all the confounding variables were basically the same between two groups. Unmarried status was still an independent risk factor for both CSS (HR: 1.231, 95\% CI: 1.137-1.332, $P<0.001$ ) and OS (HR: 1.279, 95\% CI: 1.203-1.360, $P<0.001$ ). These results suggested that marital status-related spousal support was likely to improve outcomes beyond levels of diagnosis and treatment. Details on it are discussed further in the following section.

\section{DISCUSSION}

It has been confirmed that married patients possessed lower mortality rate and favored a longer life expectancy in different cancer types [8-11]. However, impact of marital status on OCSCC survival has not been fully discovered, and few of these previous studies have focused on the underlying mechanisms of the survival advantage associated with marriage. Additionally, previous research focusing on the relationship between marital status and head neck cancer outcome ignored overall survival and concentrated merely on cancer-specific survival $[12,13]$. For the first time, our study indicated that marriage had an independent beneficial influence on not only cancerspecific survival and but overall survival of OCSCC patients as well. Particularly, our research indicated that marital status could independently predict survival in most of the stage, age, treatment, gender and race subgroups and exert a greater effect on overall survival than cancerspecific survival. Furthermore, it is the first study to clarify the reasons why marriage protects OCSCC patients from mortality in depth.

It has been shown that marriage-related spousal support improved prognosis of cancer patients in many ways. Prior literatures confirmed married people tended to undergo earlier screening and obtained more adequate treatment [7, 10]. Our study also reached similar conclusions. As can be seen in our results, multiple reasons such as earlier stage at diagnosis, preference of receiving surgery contributed to the survival advantage of married OCSCC individuals. Though widowed patients in the unmarried group was generally of much higher age and showed worse prognosis in other cancer types [8, 9], it should be noted that in our study, elder age might not account for poorer prognosis of unmarried patients. When we combined those widowed with divorced and never married population as a whole unmarried category then carried out multivariate logistic analysis, age of unmarried group was no longer higher than those married.

Moreover, independent of tumor characteristics and treatment status, the persisting survival advantage 
of married patients in the 1:1 matching analysis could be hypothetically explained by socioeconomic and psychological factors. The importance of quality of life (QOL), which included both psychological and sociocultural indicators, was emphasized in head and neck cancer survival by Ringash et al, although the authors didn't link QOL with marital status directly [14]. It has been proposed that psychology, living habits, economic conditions, as well as certain biological factors were all strongly associated with marriage. As is wellknown, smoking and alcoholism have proved to be intimately correlated to etiology and prognosis of OCSCC $[2,15]$. Lindström and Stack et al. separately reported that marriage led to less nicotine intake and alcohol consumption [16, 17]. Hence, marriage was likely to help OCSCC patients give up such bad behaviors to reduce the additional harm [18]. Male and black population possessed higher rates of smoking and drinking [19, 20], consequently it could explain why marriage had a stronger effect on survival of men and black people in our study. Psychologically, married patients had easier access to solicitude or assistance from their family and
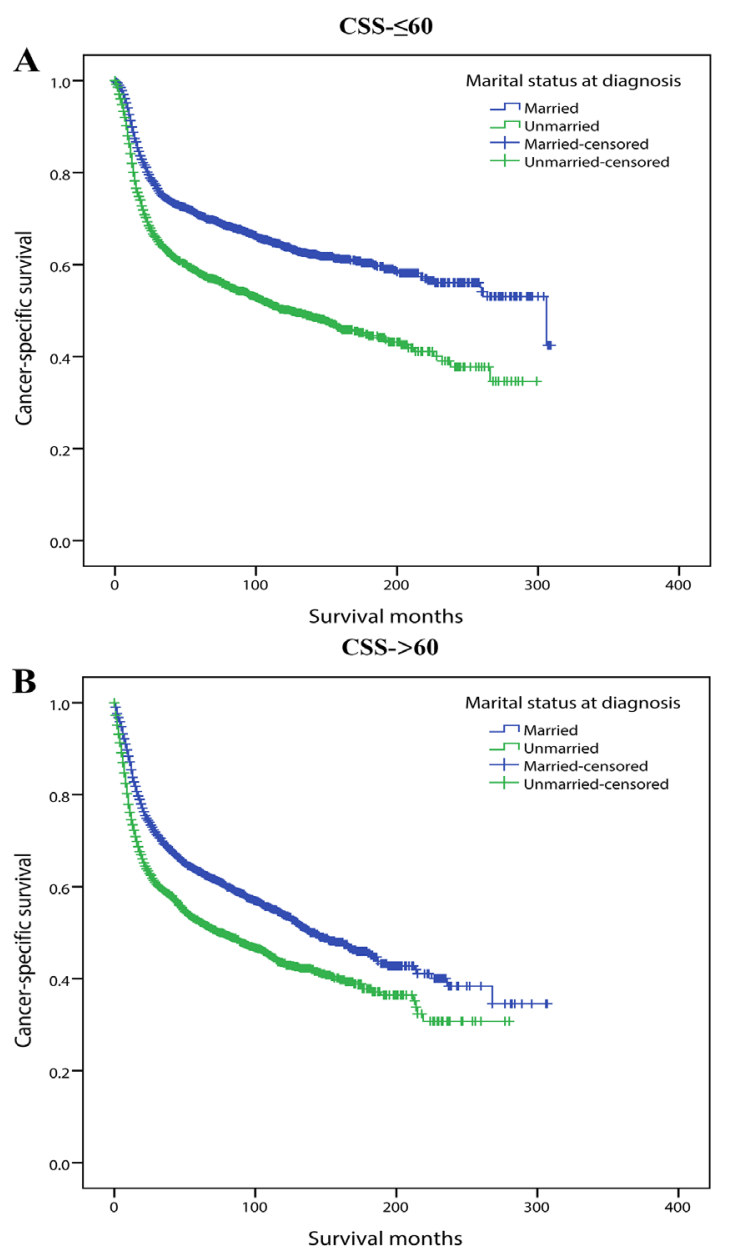

friends, and possessed higher levels of fighting spirit and lower levels of distress [21, 22]. Brown et al. reported depression symptomology was the most consistent predictor of shorten survival time in cancer patients [23]. Another research by Van der Meulen et al. also proved that head and neck cancer including OCC patients benefited from psychological interventions [24]. Therefore, more attention should be paid to psychological problems of unmarried OCSCC patients. Additionally, married people tended to have better economic status and were more likely to be insured, which can provide them better treatment and nursing conditions $[25,26]$. Physiologically, cortisol level and immunity indicators like NK cell activity, T-cell infiltration ability were all associated with distress and depression. They could be improved by spousal support more or less [27-30].

Not only cancer, marriage also played a positive role in overall health. Kubzansky reported marriage protected people from type 2 diabetes through favorable changes in lifestyle [31]. Another study of African Americans with heart failure proved that being married and living with family independently predict lower mortality and fewer
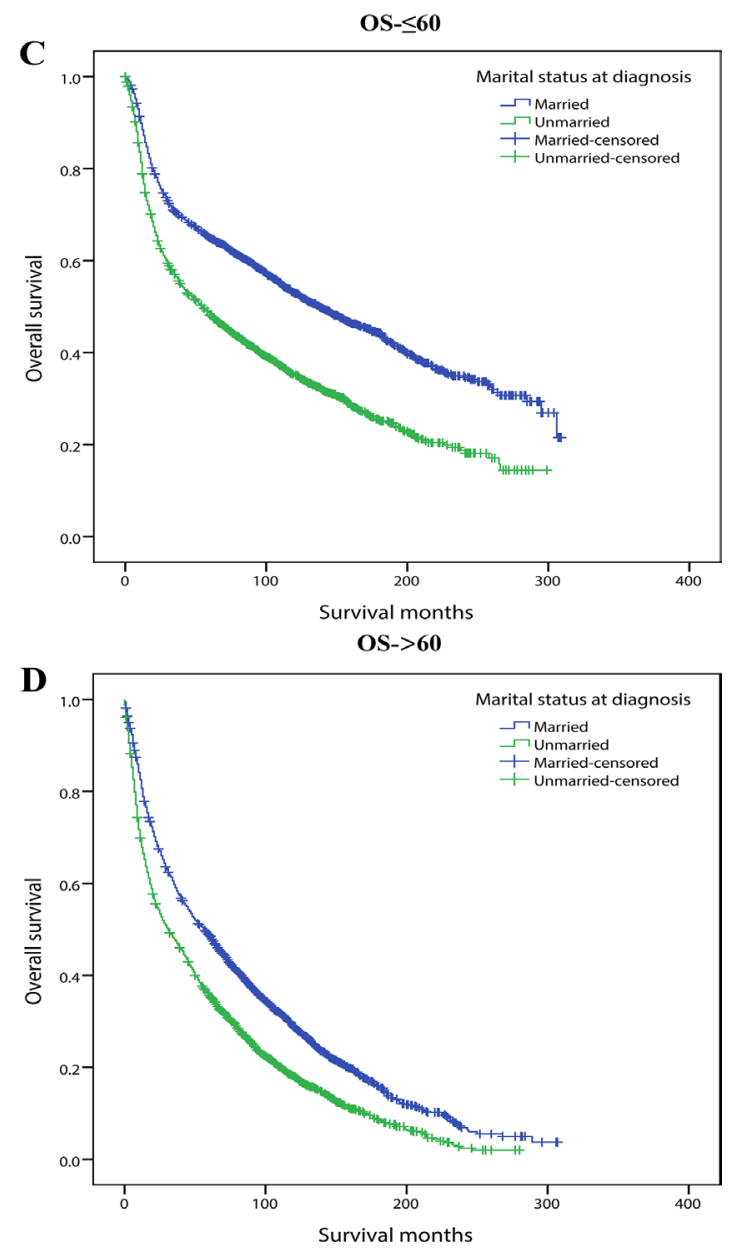

Figure 3: Kaplan-Meier survival curves: cancer-specific survival and overall survival in 11022 OCSCC patients according to $\leq \mathbf{6 0}$ and $>\mathbf{6 0}$ binary age subgroups. (A) CSS $-\leq 60: \chi^{2}=104.243, P<0.001 ;(\mathbf{B})$ CSS- $>60: \chi^{2}=80.789, P<0.001 ;(\mathbf{C})$ OS- $\leq 60: \chi^{2}=184.233, P<0.001 ;\left(\right.$ D) OS- $>60: \chi^{2}=146.355, P<0.001$. 
readmissions [32]. Besides, a research of East Asian populations revealed marriage and marital satisfaction was of great importance in determining self-rated health [33]. These studies were compatible with our results that the greater impact of marital status on OS than CSS.
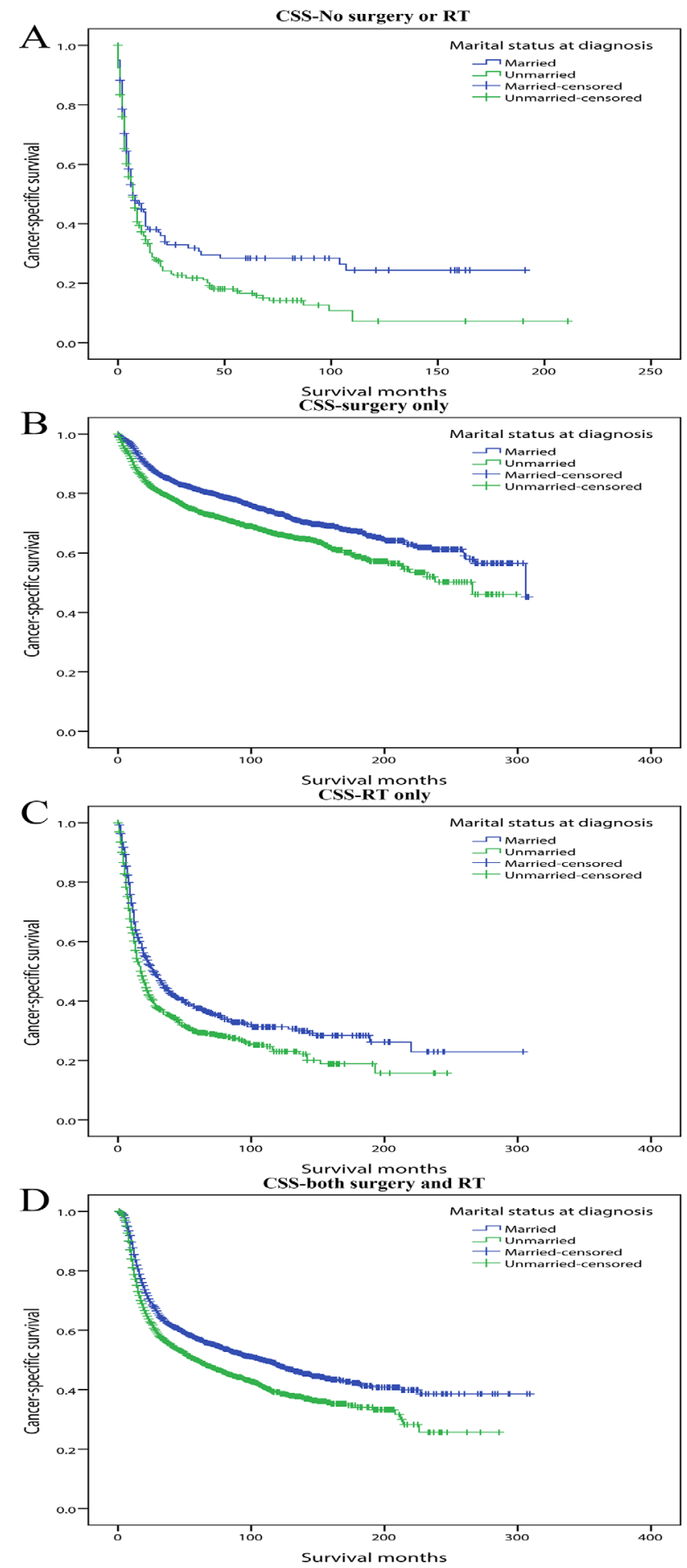

The findings of our study could help clinicians understand more about the role of marital status in prognosis of oral cavity cancer, thus allow them to pay more attention to social support of unmarried patients. For example, when a man demonstrating a higher risk of oral
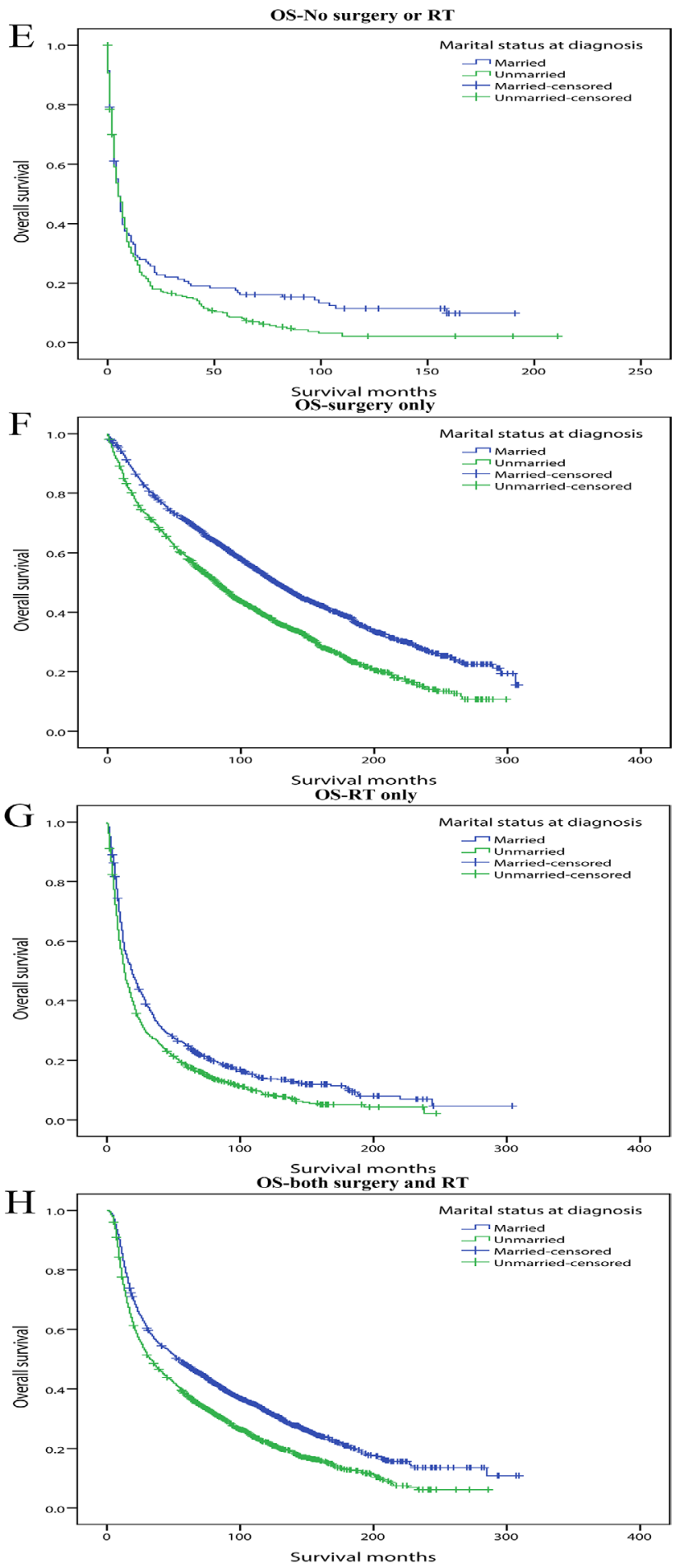

Figure 4: Kaplan-Meier survival curves: cancer-specific survival and overall survival in 11022 OCSCC patients according to treatment. (A) CSS-No surgery or RT: $\chi^{2}=5.400, P=0.020$; (B) CSS-Surgery only: $\chi^{2}=35.985, P<0.001$; (C) CSS-RT only: $\chi^{2}=14.550, P<0.001$; (D) CSS-Both surgery and RT: $\chi^{2}=31.287, P<0.001$. (E) OS-No surgery or RT: $\chi^{2}=4.389, P=0.036$; $(\mathbf{F})$ OS-Surgery only: $\chi^{2}=116.459, P<0.001 ;(\mathbf{G})$ OS-RT only: $\chi^{2}=19.604, P<0.001 ;\left(\right.$ H) OS-Both surgery and RT: $\chi^{2}=62.052, P<0.001$. 
cavity cancer came to hospital, we could leave his contact information. If he is married, we can tell his spouse to focus on oral cavity condition of the patient and remind him not to neglect his potential illness. In other words, the spouse could take on partial responsibility of social support, and thereby reduces the burden on the social health network and family physicians. However, if the patient is unmarried, he lacks the spousal support which can offer health recommendations. Utilizing the community health network or care of the family doctor, compared to a married patient, we need to remind him more frequently to pay attention to the condition of oral cavity and take examinations routinely. By providing social support in this way, we can replace the role of the spouse to some extent.

A

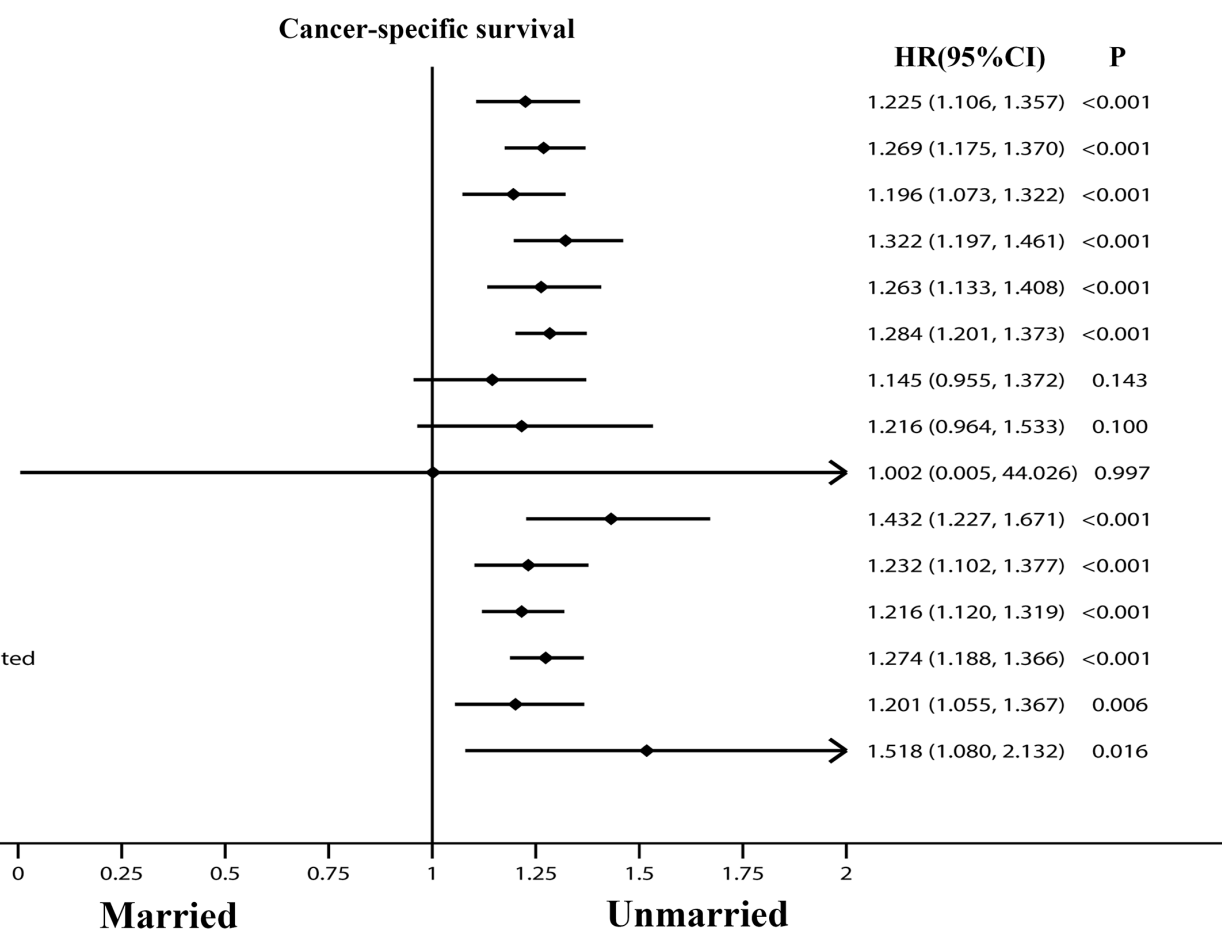

B

$\begin{aligned} \text { Gender } & \text { Female } \\ & \text { Male } \\ \text { Site } & \text { Tongue } \\ & \text { Gum and other mouth } \\ & \text { Floor of mouth } \\ \text { Race } & \text { White } \\ & \text { Black } \\ & \text { Other } \\ & \text { Unknown race } \\ \text { Year } & 1988-1994 \\ & 1995-2001 \\ & 2002-2008\end{aligned}$

Grade Well/Moderately differentiated Poorly/Undifferentiated Unknown grade

Unmarried

\section{Overall survival}

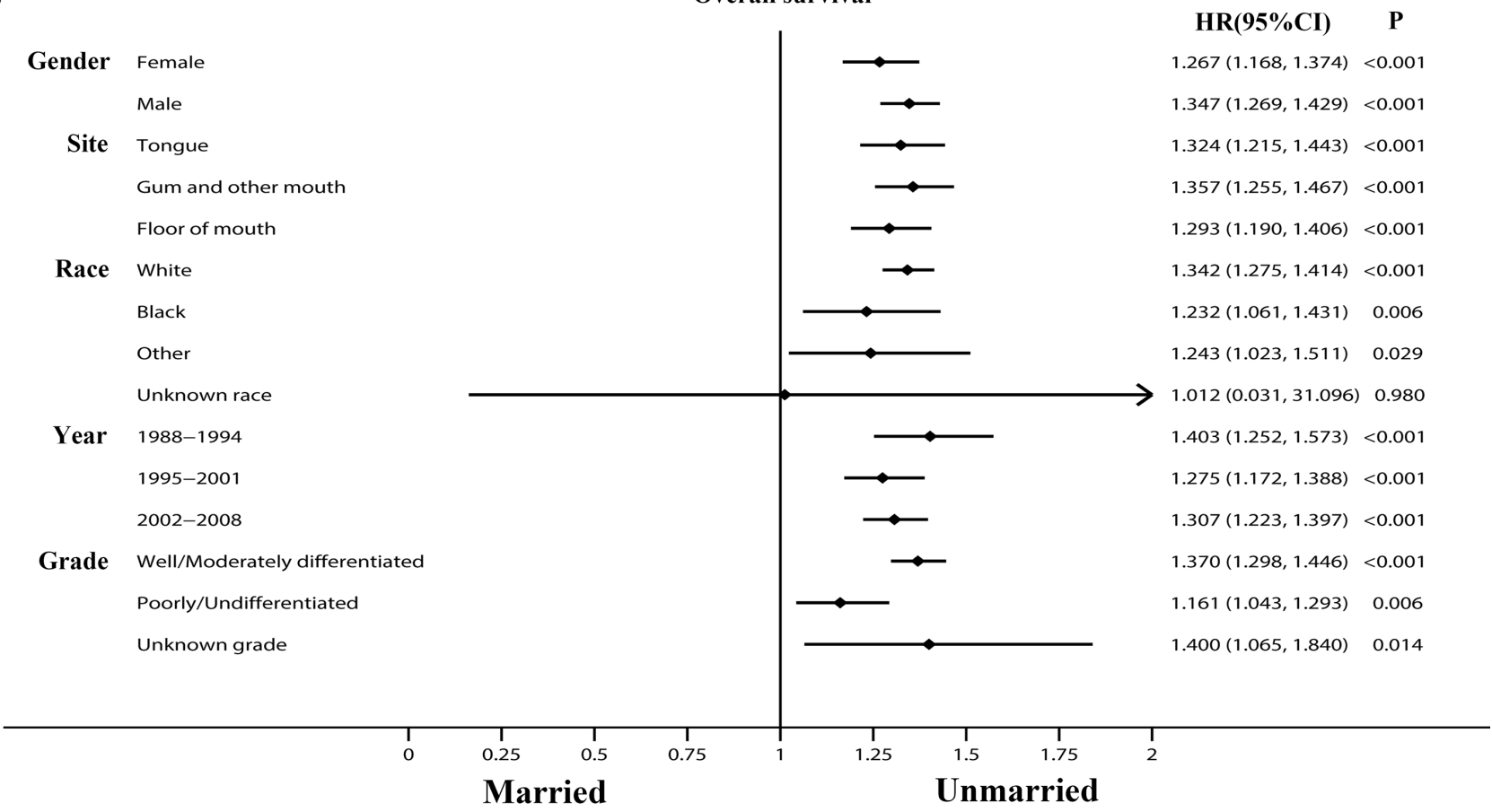

Figure 5: Forest plots summarizing hazard ratios for cancer-specific survival and overall survival in subgroup analyses: Married versus Unmarried. The X-axis displays the hazard ratio and 95\% CI of each subgroup, ticks are arranged at 0, 0.25, 0.5, 0.75, 1.0, 1.25, 1.5, 1.75, and 2.0. (A) Cancer-specific survival. (B) Overall survival. 
Table 7: Characteristics of patients by marital status in 1:1 matched group

\begin{tabular}{|c|c|c|c|c|}
\hline \multirow{2}{*}{ Characteristic } & Total & Married & Unmarried & \multirow{2}{*}{$P$} \\
\hline & $6208(100)$ & 3104 (100) & 3104 (100) & \\
\hline Gender & & & & 0.854 \\
\hline Female & $2297(37.0)$ & $1152(37.1)$ & $1145(36.9)$ & \\
\hline Male & $3911(63.0)$ & $1952(62.9)$ & $1959(63.1)$ & \\
\hline Age & & & & 1 \\
\hline$<35$ & $108(1.7)$ & $54(1.7)$ & $54(1.7)$ & \\
\hline $35-44$ & $370(6.0)$ & $185(6.0)$ & $185(6.0)$ & \\
\hline $45-54$ & $1234(19.9)$ & $617(19.9)$ & 617 (19.9) & \\
\hline $55-64$ & $1790(28.8)$ & $895(28.8)$ & $895(28.8)$ & \\
\hline $65-74$ & $1532(24.7)$ & $766(24.7)$ & $766(24.7)$ & \\
\hline $75-84$ & $980(15.8)$ & $490(15.8)$ & $490(15.8)$ & \\
\hline$>85$ & $194(3.1)$ & $97(3.1)$ & $97(3.1)$ & \\
\hline ICD-O-3 site code & & & & 0.976 \\
\hline Tongue & $2188(35.2)$ & $1090(35.1)$ & $1098(35.4)$ & \\
\hline Gum and other mouth & $2176(35.1)$ & $1091(35.1)$ & $1085(34.9)$ & \\
\hline Floor of mouth & $1844(29.7)$ & $923(29.7)$ & $921(29.7)$ & \\
\hline Race & & & & 0.893 \\
\hline White & $5451(87.8)$ & $2735(88.1)$ & $2716(87.5)$ & \\
\hline Black & $362(5.8)$ & $178(5.7)$ & $184(5.9)$ & \\
\hline Other & $377(6.1)$ & $182(5.9)$ & $195(6.3)$ & \\
\hline Unknown & $18(0.3)$ & $9(0.3)$ & $9(0.3)$ & \\
\hline Grade & & & & 1 \\
\hline Well/Moderately differentiated & $5230(84.2)$ & $2615(84.2)$ & $2615(84.2)$ & \\
\hline Poorly/Undifferentiated & $872(14.1)$ & $436(14.1)$ & $436(14.1)$ & \\
\hline Unknown & $106(1.7)$ & $53(1.7)$ & $53(1.7)$ & \\
\hline Year & & & & 0.295 \\
\hline 1988-1994 & $751(12.1)$ & $381(12.3)$ & $370(11.9)$ & \\
\hline 1995-2001 & $1557(25.1)$ & $802(25.8)$ & $755(24.3)$ & \\
\hline $2002-2008$ & $3900(62.8)$ & $1921(61.9)$ & $1979(63.8)$ & \\
\hline TNM Stage & & & & 1 \\
\hline $\mathrm{I}$ & $2076(33.4)$ & $1038(33.4)$ & $1038(33.4)$ & \\
\hline II & $1206(19.4)$ & $603(19.4)$ & $603(19.4)$ & \\
\hline III & $622(10.0)$ & $311(10.0)$ & $311(10.0)$ & \\
\hline $\mathrm{IVa} / \mathrm{IVb}$ & $2250(36.2)$ & $1125(36.2)$ & $1125(36.2)$ & \\
\hline IVc & $54(0.9)$ & $27(0.9)$ & $27(0.9)$ & \\
\hline Surgery & & & & 1 \\
\hline No surgery & $890(14.3)$ & $445(14.3)$ & $445(14.3)$ & \\
\hline Local excision/destruction & $900(14.5)$ & $450(14.5)$ & $450(14.5)$ & \\
\hline Wide/Radical excision & $4418(71.2)$ & $2209(71.2)$ & $2209(71.2)$ & \\
\hline Radiotherapy & & & & 1 \\
\hline No & $3042(49.0)$ & $1521(49.0)$ & $1521(49.0)$ & \\
\hline Yes & $3166(51.0)$ & $1583(51.0)$ & $1583(51.0)$ & \\
\hline
\end{tabular}

SEER 1988-2008 $(n=6208)$.

$P$ value was analyzed by Pearson $\chi^{2}$ test. 
Inevitably, potential limitations of our research should be taken into consideration. Firstly, in SEER database, information of some important therapies was not accessible, such as chemotherapy and biotherapy. Secondly, smoking, alcohol and HPV status were important risk and potential prognostic factors for OCSCC, marriage may also perform its protective role through less nicotine and alcohol intake, just as we've discussed above. However, these factors were not recorded in SEER database either. This limitation might lead to potential bias. Thirdly, duration of marriage could possibly affect the effect of marital status as well. But SEER database only collected marital status at the time of diagnosis, numerous patients enrolled in our study survived a long time, during the follow-up period, marital status might change and affect survival. Besides, marital satisfaction records were lacking, which was a crucial component of marital quality. Fourthly, some important etiologic factors were not recorded in SEER like tobacco and alcohol use. Finally, factors like education and income status were also likely to play a role. Ansell et al. and Boyd et al. separately proved that higher income was associated with lower cancer incidence and better prognosis, more exposure to carcinogens and lower rate of insurance were considered to be possible explanations [34-35]. Two studies based on large databases in Sweden and Finland reported that higher education level helped to achieve higher socioeconomic status, which was often related to earlier detection and adequate treatment, thus to improve prognosis [36-37]. Lack of educational and income status in SEER database might increase the possibilities of bias.

Despite these limitations above, our study confirmed the survival disparity of OCSCC patients related with marital status. In summary, married patients

A

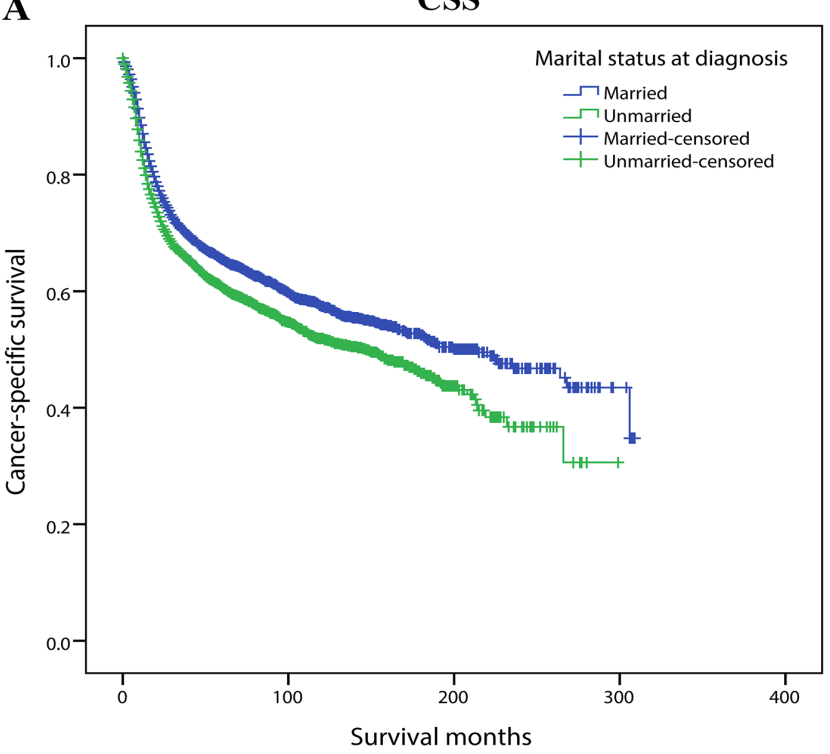

always showed a significant survival advantage than those unmarried. The results were derived from timely diagnosis, adequate treatment and probably influenced by spousal support through psychological, economical and physiological ways. Consequently, our findings heighten the awareness of the effect of marital status on OCSCC outcome and encourage clinicians to provide unmarried oral cavity cancer patients and high-risk groups with more social support, in order to help them get a better outcome, for both longer survival time and higher life quality.

\section{MATERIALS AND METHODS}

\section{Data source}

We obtained data from Surveillance, Epidemiology, and End Results (SEER) database sponsored by National Cancer Institute. SEER database collects information of cancer patients in 18 registries, covering about 28 percent of total U.S population. The database includes some important oncological data, such as demographics, primary sites, morphology, stage, surgery, radiotherapy, grade and patients' vital status. SEER*Stat 8.3.2 software was used to extract information from the database.

\section{Inclusion criteria}

The inclusion criteria were as follows: 1) Known marital status. 2) 18 years old or older at the time of diagnosis. 3) Diagnosed with OCSCC only or multiple primary cancers but OCSCC was the first. 4) The ICD-O-3 site codes were limited to C02.0-02.3, 02.8-02.9, 03.0$03.9,04.0-05.0,05.8-06.9$. It's worth noting that codes 00.0-00.9 (Lip) were not included because NCCN

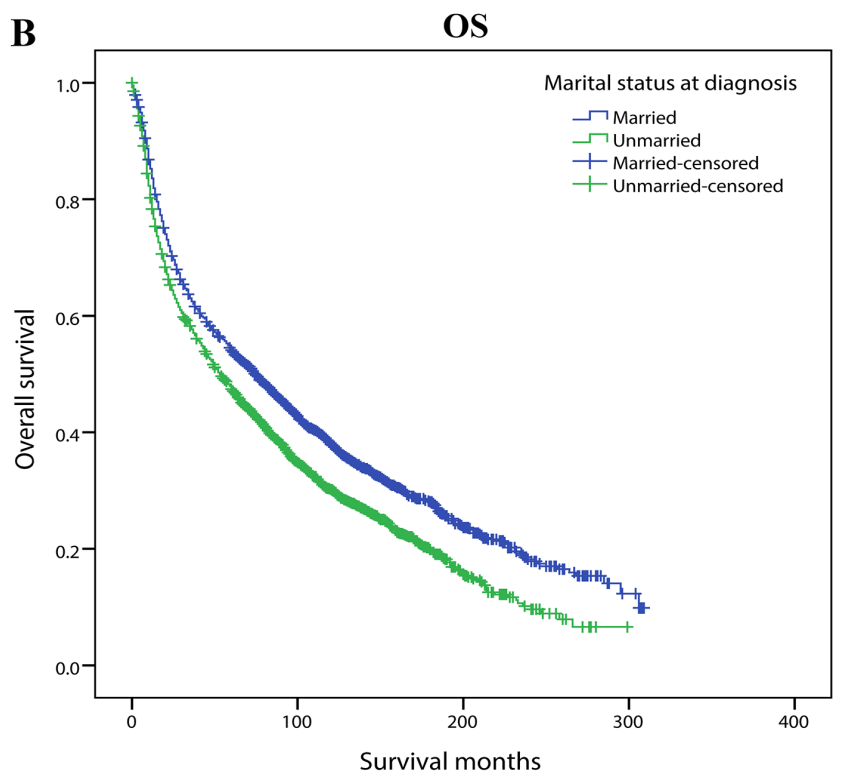

Figure 6: Kaplan-Meier survival curves of 1:1 matched group: cancer-specific survival and overall survival in 6208 OCSCC patients. Married vs Unmarried (A) Cancer-specific survival: $\chi^{2}=21.669, P<0.001$. (B) Overall survival: $\chi^{2}=49.785, P<0.001$. 
regarded lip and oral cavity as two different parts and provided different treatment guidelines respectively [38]. Subsites of oropharynx were also excluded like base of tongue, tonsil, soft palate and uvula, etc. 5) Histological type was limited to squamous cell carcinoma $(8052,8070$ $8078,8083-8084)$ according to ICD-O-3 histological codes. 6) Diagnosed between 1/1/1988 and 12/31/2008 to ensure an adequate 5 -year follow-up time, as the follow-up cutoff date of currently available SEER data was 12/31/2013. 7) Active follow-up. 8) Known survival months after diagnosis and known cause of death. 9) Known surgery condition and radiotherapy condition 10) Definite AJCC TNM stage at diagnosis.

\section{Study variables}

Patient's important characteristics were extracted from SEER database to be our study variables, including marital status, TNM stage at diagnosis, age at diagnosis, gender, primary tumor site, tumor grade, race, surgery condition and radiotherapy condition. AJCC TNM stage IV was divided into stage $\mathrm{IVa} / \mathrm{IVb}$ and stage $\mathrm{IVc}$ since stage IVc meant distant metastasis and its prognosis could be rather different from stage IVa and IVb. Marital status which was the primary variable of interest was analyzed as a bivariate value (married and unmarried).

\section{Statistical analysis}

Baseline characteristics were compared by $\chi^{2}$ test. The primary end points of our study were CSS and OS, which were analyzed by Kaplan-Meier analysis and the difference was calculated by Log-rank test. Relationship between marital status and TNM stage at diagnosis, age at diagnosis and treatment were conducted by binomial or multinomial logistic regression. Multivariate Cox proportional hazards regression models were built for presenting hazard ratios of different study variables. We also used forest plots to summarize risk factors for survival in subgroup analyses. A 1:1 paired cohort matching with marital status was performed by propensity score matching (PSM) method.

Kaplan-Meier curve, logistic regression, multivariate Cox regression and $\chi^{2}$ test were analyzed by statistical software IBM SPSS, version 22 (SPSS Inc, Chicago, IL, USA). Propensity score matching method and forest plots were conducted by Stata statistical software, version 14.0 (StataCorp, College Station, TX). All $P$ values were twosided and statistical significance was set at $P<0.05$.

\section{Authors' contributions}

$\mathrm{XS}, \mathrm{TTZ}$ and QHJ conceived and designed this study. XS, TTZ and WPH performed the analyses. XS and TTZ prepared all the tables and figures. XS wrote the main manuscript. All authors reviewed the manuscript.

\section{ACKNOWLEDGMENTS}

The authors of this study acknowledge the contribution of SEER database and the 18 registries supplying cancer research information.

\section{CONFLICTS OF INTEREST}

None of the authors have any conflicts of interest to declare.

\section{REFERENCES}

1. Torre LA, Bray F, Siegel RL, Ferlay J, Lortet-Tieulent J, Jemal A. Global cancer statistics, 2012. CA Cancer J Clin. 2015; 65:87-108.

2. Chi AC, Day TA, Neville BW. Oral cavity and oropharyngeal squamous cell carcinoma - an update. CA Cancer J Clin. 2015; 65:401-421.

3. Siegel RL, Miller KD, Jemal A. Cancer statistics, 2016. CA Cancer J Clin. 2016; 66:7-30.

4. Weatherspoon DJ, Chattopadhyay A, Boroumand S, Garcia I. Oral cavity and oropharyngeal cancer incidence trends and disparities in the United States: 2000-2010. Cancer Epidemiol. 2015; 39:497-504.

5. Elwood JM, Youlden DR, Chelimo C, Ioannides SJ, Baade PD. Comparison of oropharyngeal and oral cavity squamous cell cancer incidence and trends in New Zealand and Queensland, Australia. Cancer Epidemiol. 2014; 38:16-21.

6. Chaturvedi AK, Anderson WF, Lortet-Tieulent J, Curado MP, Ferlay J, Franceschi S, Rosenberg PS, Bray F, Gillison ML. Worldwide trends in incidence rates for oral cavity and oropharyngeal cancers. J Clin Oncol. 2013; 31:4550-4559.

7. Aizer AA, Chen MH, McCarthy EP, Mendu ML, Koo S, Wilhite TJ, Graham PL, Choueiri TK, Hoffman KE, Martin NE, Hu JC, Nguyen PL. Marital status and survival in patients with cancer. J Clin Oncol. 2013; 31:3869-3876.

8. Li Q, Gan L, Liang L, Li X, Cai S. The influence of marital status on stage at diagnosis and survival of patients with colorectal cancer. Oncotarget. 2015; 6:7339-7347. doi: 10.18632/oncotarget.3129.

9. Shi RL, Qu N, Lu ZW, Liao T, Gao Y, Ji QH. The impact of marital status at diagnosis on cancer survival in patients with differentiated thyroid cancer. Cancer Med. 2016; 5:2145-2154.

10. Zhang J, Gan L, Wu Z, Yan S, Liu X, Guo W. The influence of marital status on the stage at diagnosis, treatment, and survival of adult patients with gastric cancer: a population-based study. Oncotarget. 2016. doi: 10.18632/oncotarget.7399. [Epub ahead of print]

11. Costa LJ, Brill IK, Brown EE. Impact of marital status, insurance status, income, and race/ethnicity on the survival of younger patients diagnosed with multiple myeloma in the United States. Cancer. 2016; 122:3183-3190. 
12. Inverso G, Mahal BA, Aizer AA, Donoff RB, Chau NG, Haddad RI. Marital status and head and neck cancer outcomes. Cancer. 2015; 121:1273-1278.

13. Schaefer EW, Wilson MZ, Goldenberg D, Mackley H, Koch W, Hollenbeak CS. Effect of marriage on outcomes for elderly patients with head and neck cancer. Head Neck. 2015; 37:735-742.

14. Ringash J. Survivorship and Quality of Life in Head and Neck Cancer. J Clin Oncol. 2015; 33:3322-3327.

15. Aarstad AK, Aarstad HJ, Olofsson J. Quality of life, drinking to cope, alcohol consumption and smoking in successfully treated HNSCC patients. Acta Otolaryngol. 2007; 127:1091-1098.

16. Lindstrom M. Social capital, economic conditions, marital status and daily smoking: a population-based study. Public Health. 2010; 124:71-77.

17. Stack S, Wasserman IM. Marital status, alcohol abuse and attempted suicide: a logit model. J Addict Dis. 1995; 14:43-51.

18. Power C, Rodgers B, Hope S. Heavy alcohol consumption and marital status: disentangling the relationship in a national study of young adults. Addiction. 1999; 94:1477-1487.

19. Jamal A, Agaku IT, O'Connor E, King BA, Kenemer JB, Neff L. Current cigarette smoking among adults-United States, 2005-2013. MMWR Morb Mortal Wkly Rep. 2014; 63:1108-1112.

20. Delker E, Brown Q, Hasin DS. Alcohol Consumption in Demographic Subpopulations: An Epidemiologic Overview. Alcohol Res. 2016; 38:7-15.

21. Taniguchi K, Akechi T, Suzuki S, Mihara M, Uchitomi Y. Lack of marital support and poor psychological responses in male cancer patients. Support Care Cancer. 2003; 11:604-610.

22. Saito-Nakaya K, Nakaya N, Fujimori M, Akizuki N, Yoshikawa E, Kobayakawa M, Nagai K, Nishiwaki Y, Tsubono Y, Uchitomi Y. Marital status, social support and survival after curative resection in non-small-cell lung cancer. Cancer Sci. 2006; 97:206-213.

23. Brown KW, Levy AR, Rosberger Z, Edgar L. Psychological distress and cancer survival: a follow-up 10 years after diagnosis. Psychosom Med. 2003; 65:636-643.

24. Van der Meulen IC, May AM, de Leeuw JR, Koole R, Oosterom M, Hordijk GJ, Ros WJ. Long-term effect of a nurse-led psychosocial intervention on health-related quality of life in patients with head and neck cancer: a randomised controlled trial. Br J Cancer. 2014; 110:593-601.

25. Byers TE, Wolf HJ, Bauer KR, Bolick-Aldrich S, Chen VW, Finch JL, Fulton JP, Schymura MJ, Shen T, Van Heest S, Yin X. The impact of socioeconomic status on survival after cancer in the United States : findings from the National Program of Cancer Registries Patterns of Care Study. Cancer. 2008; 113:582-591.

26. Grant SR, Walker GV, Guadagnolo BA, Koshy M, Allen PK, Mahmood U. Variation in insurance status by patient demographics and tumor site among nonelderly adult patients with cancer. Cancer. 2015; 121:2020-2028.
27. Sapolsky RM, Romero LM, Munck AU. How do glucocorticoids influence stress responses? Integrating permissive, suppressive, stimulatory, and preparative actions. Endocr Rev. 2000; 21:55-89.

28. Antoni MH, Cruess S, Cruess DG, Kumar M, Lutgendorf S, Ironson G, Dettmer E, Williams J, Klimas N, Fletcher MA, Schneiderman N. Cognitive-behavioral stress management reduces distress and 24-hour urinary free cortisol output among symptomatic HIV-infected gay men. Ann Behav Med. 2000; 22:29-37.

29. Levy SM, Herberman RB, Whiteside T, Sanzo K, Lee J, Kirkwood J. Perceived social support and tumor estrogen/ progesterone receptor status as predictors of natural killer cell activity in breast cancer patients. Psychosom Med. 1990; 52:73-85.

30. Dhabhar FS. Effects of stress on immune function: the good, the bad, and the beautiful. Immunol Res. 2014; 58:193-210.

31. Cornelis MC, Chiuve SE, Glymour MM, Chang SC, Tchetgen Tchetgen EJ, Liang L, Koenen KC, Rimm EB, Kawachi I, Kubzansky LD. Bachelors, divorcees, and widowers: does marriage protect men from type 2 diabetes? PLoS One. 2014; 9:e106720.

32. Lu ML, Davila CD, Shah M, Wheeler DS, Ziccardi MR, Banerji S, Figueredo VM. Marital status and living condition as predictors of mortality and readmissions among African Americans with heart failure. Int J Cardiol. 2016; 222:313-318.

33. Chung W, Kim R. Does marriage really matter to health? Intra- and inter-country evidence from China, Japan, Taiwan, and the Republic of Korea. PLoS One. 2014; 9:e104868.

34. Ansell D, Whitman S, Lipton R, Cooper R. Race, income, and survival from breast cancer at two public hospitals. Cancer. 1993; 72:2974-2978.

35. Boyd C, Zhang-Salomons JY, Groome PA, Mackillop WJ. Associations between community income and cancer survival in Ontario, Canada, and the United States. J Clin Oncol. 1999; 17:2244-2255.

36. Pokhrel A, Martikainen P, Pukkala E, Rautalahti M, Seppa K, Hakulinen T. Education, survival and avoidable deaths in cancer patients in Finland. Br J Cancer. 2010; 103:1109-1114.

37. Hussain SK, Lenner P, Sundquist J, Hemminki K. Influence of education level on cancer survival in Sweden. Ann Oncol. 2008; 19:156-162.

38. National Comprehensive Cancer Network. NCCN Guidelines. Head and Neck Cancer. Version 1, 2016. Available at: https://www.ncen. org/professionals/physician_gls/pdf/head-and-neck_blocks.pdf. 